\title{
THE WEIGHT OF A PSEUDOCOMPACT (HOMOGENEOUS) SPACE WHOSE CARDINALITY HAS COUNTABLE COFINALITY
}

\author{
ERIC K. VAN DOUWEN ${ }^{1}$
}

\begin{abstract}
Let $X$ be an infinite pseudocompact space. We are interested in restrictions on $\kappa=|X|$ and $\lambda=w(X)$ in addition to the obvious inequalities $\lambda<2^{\kappa}$ and $\kappa<2^{\lambda}$, and $\kappa>2^{\omega}$, valid for $X$ without isolated points (in particular for homogeneous $X$ ). We show that if $\operatorname{cf}(\kappa)=\omega$, then $\lambda<2^{<\kappa}$, and even $\lambda<2^{\mu}$ for some $\mu<\kappa$ if $X$ is homogeneous. Under the Singular Cardinals Hypothesis (which is much weaker than the GCH), there are no further restrictions for $X$ without isolated points.
\end{abstract}

0. Conventions. All spaces considered are completely regular, and "group" means "topological group". As usual, $\mathrm{d}, \mathrm{nw}, \mathrm{w}$ and $\chi$ denote the cardinal functions density, network weight, weight and character (note that we do not use character in the group theoretic sense). All unexplained inequalities involving cardinal functions are generally valid and have easy proofs, cf. [En, §1.5] or [Ju, p. 9.10].

We use $\kappa, \lambda, \mu$ for infinite cardinals (= initial ordinals). For typographical reasons we often use $\exp \kappa$ for $2^{\kappa}, \exp ^{2} \kappa$ for $2^{2^{k}}$ and

$$
\exp _{<} \kappa \text { for } 2^{<\kappa}=\sup \left\{2^{\gamma}: \gamma \text { a cardinal, } \gamma<\kappa\right\} \text {. }
$$

Recall that $\kappa$ is called a strong limit if $2^{\gamma}<\kappa$ for each cardinal $\gamma<\kappa$. For all $\lambda$ and $\mu$ with $\mu$ regular there is a strong limit $\kappa$ with $\kappa>\lambda$ and $\operatorname{cf}(\mu)=\omega$. This tells that Corollary 1.2 below is nonvacuous.

1. Results. Hodel has asked if for each $\kappa>\omega$ there is a countably compact space $X$ with $\mathrm{w}(X)>|X|=\kappa,[\mathbf{H}, 3.4]$; this question is motivated by the fact that $\mathrm{w}(X)<|X|$ if $X$ is compact, [En, 3.1.21]. As mentioned by Hodel, [H, 3.4], Comfort has shown that the answer is yes for $\kappa$ satisfying $\kappa^{\omega}=\kappa$ (see §3). We will show that the answer is no for certain other $\kappa$, but can be yes for $\kappa$ with $\kappa^{\omega} \neq \kappa$, see §4. In fact we investigate the more general question for which $\kappa$ and $\lambda$ there is a space $X$ with $|X|=\kappa$ and $w(X)=\lambda$ which is pseudocompact, or pseudocompact and homogeneous.

The two inequalities restricting weight and cardinality of a space $X$ are

$$
w(X)<\exp |X| \text { and }|X|<\exp w(X) .
$$

\footnotetext{
Received by the editors September 20, 1979 and, in revised form, January 21, 1980.

1980 Mathematics Subject Classification. Primary 54A25, 54D30, 54C99, 54E65; Secondary 54A35, 54D35.

Key words and phrases. Weight, cardinality, countably cofinality, strong limit, pseudocompact, homogeneous.

'Research supported by NSF Grant MCS 78-09484.
} 
This is the only restriction on $w(X)$ and $|X|$ : for all $\kappa$ and $\lambda$ with $\lambda<2^{\kappa}$ and $\kappa<2^{\lambda}$ one can find a space $X$ (which can be taken to be a group, see \$3) with $|X|=\kappa$ and $\mathbf{w}(X)=\lambda$.

[Note that the statement that (*) gives the only restriction on $w(X)$ and $|X|$ is formally stronger than the statement that the inequalities in (*) are sharp.]

For pseudocompact (homogeneous) $X$ we find an additional restriction.

1.1 THEOREM. Let $X$ be a space with $\operatorname{cf}(|X|)=\omega$.

(A) If $X$ is pseudocompact then $w(X)<\exp _{<}|X|$.

(B) If $X$ is pseudocompact and homogeneous then $\mathrm{d}(X)<|X|$, hence $\mathrm{w}(X)<2^{\mu}$ for some $\mu<|X|$.

1.2 COROLlary. If $X$ is pseudocompact, and if $|X|$ is a strong limit with $\operatorname{cf}(|X|)=$ $\omega$, then $\mathrm{w}(X)=|X|$ and $X$ is not homogeneous.

There is another, elementary, additional restriction, which should be known.

1.3 Proposition. Let $X$ be pseudocompact.

(a) If $X$ has no isolated points, then $|X|>2^{\omega}$.

(b) If $X$ is infinite and homogeneous, then $X$ has no isolated points.

Under a mild set theoretic assumption there are no other additional restrictions on the weight and cardinality of a pseudocompact (even: countably compact) space without isolated points, or on the weight and cardinality of a pseudocompact homogeneous space (even: group).

1.4 EXAMPLE. Assume the following statement

$$
\text { if } \mu>2^{\omega} \text { and } \operatorname{cf}(\mu) \neq \omega \text {, then } \mu^{\omega}=\mu \text {. }
$$

Let $\kappa, \lambda$ be cardinals with $2^{\omega}<\kappa<2^{\lambda}$ and $\lambda<2^{\kappa}$.

(A) If also $\lambda<2^{<\kappa}$ in case $\operatorname{cf}(\kappa)=\omega$, then there is a countably compact space $X$ without isolated points satisfying $|X|=\kappa$ and $\mathrm{w}(X)=\lambda$.

(B) If also $\lambda<2^{\mu}$ for some $\mu<\kappa$ in case $\mathrm{cf}(\kappa)=\omega$, then there is a pseudocompact group $X$ with $|X|=\kappa$ and $\mathrm{w}(X)=\lambda$.

For pseudocompact $X$ with $|X|<2^{\omega}$ there is at least one more additional restriction: $|X|=\omega$ iff $\mathrm{w}(X)=\omega$; I did not investigate the question of whether there are any further additional restrictions for such $X$. A more interesting question is whether 1.1(B) and 1.3 are best possible for countably compact groups. An attractive possibility is suggested in this question.

1.5 QUESTION. If $X$ is an infinite group (or homogeneous space) which is countably compact, is $|X|^{\omega}=|X|$ ? Is at least $\operatorname{cf}(|X|) \neq \omega$ ?

By 1.2 the answer is yes under the GCH. If the answer (to the first question in $1.5)$ is yes, then there are no other restrictions, as will become clear in $\$ 3$.

Another question is whether ( $\dagger$ ) is needed for Example 1.4. We here point out that $(\dagger)$ is much weaker than the $\mathrm{GCH}$ : Easton has shown that any "rule" that is not obviously false can be used to tell what $2^{\kappa}$ is for regular $\kappa$, and his models satisfy ( $\dagger$, [E]. Magidor has shown that the consistency of the existence of large cardinals implies the consistency of ZFC $+\neg(\dagger)$, and mentions that Dodd and 
Jensen have shown that one needs a large cardinal, $\left[\mathbf{M}_{1}\right],\left[\mathbf{M}_{2}\right]$. (By contrast: the consistency of $\mathrm{ZFC}+\neg \mathrm{GCH}$ follows from the consistency of $\mathrm{ZFC}$ alone.) We also point out that $(\dagger)$ is known to be equivalent to the so-called Singular Cardinals Hypothesis (i.e. for all singular (whether $\operatorname{cf}(\kappa)=\omega$ or not), if $\kappa>2^{\mathrm{cf}(\kappa)}$ then $\left.\kappa^{\mathrm{cf}(\kappa)}=\kappa^{+}\right),[\mathbf{J}, \S 8]$.

\section{Proofs.}

2.1 Proof of TheOrem 1.1. It is well known that $X$ is pseudocompact iff

(1) every nonempty $G_{\delta}$ in $\beta X$ intersects $X$, [En, 3.10E], [GJ, 6I], iff

(2) for every countable open cover $\mathscr{U}$ of $X$ there is a finite $\mathscr{F} \subseteq \mathscr{U}$ with $X=\mathbf{C l}_{\boldsymbol{X}} \cup \mathcal{F},[\mathbf{B C M}, \mathbf{1}(\mathrm{iv})]$.

Since $\operatorname{cf}(|X|)=\omega$, there is a countable collection of subsets of $X$ with $X=\cup \mathbb{Q}$ and $|A|<|X|$ for $A \in \mathbb{Q}$, and there is a countable set $M$ of cardinals with $\sup (M)=|X|$ and $\mu<|X|$ for $\mu \in M$.

We prove (A): From (1) we see that $\beta X=\cup_{A \in Q} \mathrm{Cl}_{\beta X} A$. As $\mathrm{w}\left(\mathrm{Cl}_{\beta X} A\right)<$ $\exp \mathrm{d}\left(\mathrm{Cl}_{\beta X} A\right) \leqslant \exp |A|$ for $A \in \mathbb{Q}$, it follows that the infinite compact space $\beta X$ is the union of a countable collection of subspaces each of weight at most $\exp _{<}|X|$. Hence $w(\beta X)<\exp _{<}|X|,\left[\right.$ En, 3.1.20] (for clearly $\operatorname{nw}(\beta X)<\exp _{<}|X|$, and $w(\beta X)$ $=\operatorname{nw}(\beta X)$ since $\beta X$ is compact, [En, 3.1.19]).

We prove (B): Clearly (1) implies that $X$ is a Baire space, hence there is $B \in \mathbb{Q}$ with Int ${ }_{X} \mathrm{Cl}_{X} B \neq \varnothing$. As $\mathrm{d}\left(\operatorname{Int}_{X} \mathrm{Cl}_{X} B\right)<|B|$, and $X$ is homogeneous, it follows that

(3) each $x \in X$ has a neighborhood $U_{x}$ with $\mathrm{d}\left(U_{x}\right)<|X|$.

[Actually $\mathrm{d}\left(U_{x}\right)<|B|<|X|$ for all $x \in X$. We use the weaker statement (3) since it yields an interesting Corollary to Proof.] For $A \in \mathbb{Q}$ and $\mu \in M$ define

$$
V_{A, \mu}=\cup\left\{U_{x}: x \in A, \mathrm{~d}\left(U_{x}\right)<\mu\right\} \text {. }
$$

Then $\mathscr{V}=\left\{V_{A, \mu}: A \in \mathbb{Q}, \mu \in M\right\}$ is a countable open cover of $X$. By (2) there is a finite $\mathscr{F} \subseteq \mathscr{V}$ with $X=\mathrm{Cl}_{X} \cup \mathscr{F}$. Clearly $\mathrm{d}(\cup \mathscr{F})<|X|$. Hence $\mathrm{d}(X)<|X|$.

2.2 Corollary to Proof. If $X$ is pseudocompact, and if $|X|$ is a strong limit with $\operatorname{cf}(|X|)=\omega$, then some but not all points of $X$ have a neighborhood of cardinality strictly less than $|X|$.

Since $X$ is a strong limit and $|U|<\exp ^{2} \mathrm{~d}(U)$, the inequalities $|U|<|X|$ and $\mathrm{d}(U)<|X|$ are equivalent (for neighborhoods $U$ ).

2.3 Corollary to Proof. If $X$ is a homogeneous Baire space with $\operatorname{cf}(|X|=\omega)$, then $\mathrm{w}(X)<|X| \cdot 2^{\mu}$ for some $\mu<|X|$.

2.4 Proof of Corollary 1.2. Note that $|X|<\exp w(X)$, hence $|X|<\mathrm{w}(X)$ since $|X|$ is a strong limit.

2.5 Proof of Proposition 1.3A. Apply (1) of 2.1 and an obvious Cantor tree argument.

3. The examples. Let $\Pi$ be the product of $\lambda$ factors $\{0,1\}$. Then $\Pi$ is a compact group under coordinatewise addition modulo 2 . If $D \subseteq \Pi$ is dense and $x \in D$ then $\chi(x, D)=\chi(x, \Pi)=\lambda$. As $\chi(D)<\mathrm{w}(D)<\mathrm{w}(\Pi)=\lambda$ it follows that $\mathrm{w}(D)=\lambda$. Consequently all examples below have weight $\lambda$. 
First assume only $\kappa<2^{\lambda}$ and $\lambda<2^{\kappa}$. Then $\mathrm{d}(\Pi)<\kappa$ since $\lambda<2^{\kappa}$, [En, 2.3.15], and $\kappa<|\Pi|$, hence $\Pi$ has a dense subgroup $G$ of cardinality $\kappa$, namely the subgroup generated by some dense set of cardinality $\kappa$. (This justifies the statement in the introduction that (*) is the only limitation even for groups.)

Now assume also that $\kappa^{\omega}=\kappa$. It is easy to find subgroups $G_{\alpha}\left(\alpha<\omega_{1}\right)$ with $G \subseteq G_{\alpha} \subseteq G_{\beta}\left(\alpha<\beta<\omega_{1}\right)$, and $\left|G_{\alpha}\right|=\kappa$ and each countable set in $G_{\alpha}$ has a cluster point in $G_{\alpha+1}$. Then $X=\cup_{\alpha} G_{\alpha}$ is a dense countably compact subgroup of $\Pi$ with $|X|=\kappa$. (This construction is taken from [SS, 4.5], and goes back to [F, 2.9].)

Next, assume $\kappa^{\omega} \neq \kappa>2^{\omega}$, but there is $\mu<\kappa$ (hence $\mu<2^{\lambda}$ ) with $\lambda<2^{\mu}$. As $\left(\mu^{+}\right)^{\omega}=\mu^{+}$, by $(\dagger)$, and since $\kappa>2^{\omega}$, we may assume $\mu^{\omega}=\mu$. We have just seen that $\Pi$ has a dense countably compact subgroup $H$ with $|H|=\mu$. There is a subgroup $X$ of $\Pi$ with $X \supseteq H$ and $|X|=\kappa$ (since $\mu<\kappa<|\Pi|$ ). Then $X$ is pseudocompact since it has a dense countably compact subgroup.

Finally, assume $\kappa^{\omega} \neq \kappa \geqslant 2^{\omega}$, and $\lambda \leqslant 2^{<\kappa}$, but there is no $\mu<\kappa$ with $\lambda<2^{\mu}$ (so that $\left.\lambda=2^{<\kappa}\right)$. Since we assume $(\dagger)$ and since $\kappa>2^{\omega}$, there is a countable set $M$ of cardinals with $\kappa=\sup (M)$ and $\mu^{\omega}=\mu$ for $\mu \in M$. For $\mu \in M$ let $Y_{\mu}$ be a countably compact group with $\left|Y_{\mu}\right|=\mu$ and $\mathrm{w}\left(Y_{\mu}\right)=2^{\mu}$. Let $X$ be space obtained from the topological sum of the $Y_{\mu}$ 's by adjunction of a point neighborhoods of which include all but finitely many $Y_{\mu}$ 's. Then $X$ is a countably compact space without isolated points such that $|X|=\kappa$ and $\mathrm{w}(X)=2^{<\kappa}=\lambda$.

4. Illustrations. We illustrate the results of this paper with two examples, neither of which requires $(\dagger)$, as is clear from an analysis of $\$ 3$.

First, let $\kappa$ be a strong limit with $\operatorname{cf}(\kappa)=\omega$. Then there is an $X$ with $|X|=\kappa$ and $\mathrm{w}(X)=\lambda$ such that

$\begin{array}{ll}X \text { is any space } & \text { iff } \kappa<\lambda<2^{\kappa}, \\ X \text { is any group } & \text { iff } \kappa<\lambda<2^{\kappa}, \\ X \text { is countably compact (or pseudocompact) } & \text { iff } \lambda=\kappa, \\ X \text { is a pseudocompact group } & \text { for no } \lambda .\end{array}$

Next, by Easton's results, [E], it is consistent with ZFC that

$$
2^{\omega}=\omega_{81} \text {, and } \exp \omega_{n}=\omega_{\omega+n}(1<n<\omega) \text {, and } \exp \omega_{\omega}=\omega_{\omega+\omega+1} \text {. }
$$

If we assume this, then there is an $X$ with $|X|=\omega_{\omega}$ and $w(X)=\lambda$ such that
$X$ is any space
iff $\omega_{1}<\lambda<\omega_{\omega+\omega+1}$,
$X$ is any group
$X$ is countably compact (or pseudocompact)
iff $\omega_{1}<\lambda<\omega_{\omega+\omega+1}$,
$X$ is a pseudocompact group
iff $\omega_{1}<\lambda<\omega_{\omega+\omega}$,
iff $\omega_{1}<\lambda<\omega_{\omega+\omega}$.

\section{REFERENCES}

[BCM] R. W. Bagley, E. H. Connell and J. D. McKnight, On properties characterizing pseudocompact spaces, Proc. Amer. Math. Soc. 9 (1958), 500-506.

[E] W. B. Easton, Powers of regular cardinals, Ann. Math. Logic 1 (1970), 139-170.

[En] R. Engelking, General topology, PWN, Warsaw, 1977. 
[F] Z. Frolik, The topological product of two pseudocompact spaces, Czechoslovak Math. J. 10 (85) (1960), 329-338.

[G] L. Gillman and M. Jerison, Rings of continuous functions, Van Nostrand, Princeton, N. J., 1960.

[H] R. E. Hodel, On the weight of a topological space, Proc. Amer. Math. Soc. 43 (1974), 470-474.

[J] T. Jech, Set theory, Academic Press, New York, 1978.

[Ju] I. Juhász, Cardinal functions in topology, Math. Centrum Tracts, no. 34, Math. Centrum, Amsterdam, 1971.

[M] M. Magidor, On the singular cardinals problem. I, Israel J. Math. 28 (1977), 1-33.

$\left[\mathrm{M}_{2}\right]$, On the singular cardinals problem. II, Ann. of Math. (2) 106 (1977), 517-547.

[SS] V. Saks and R. M. Stephenson, Products of m-compact spaces, Proc. Amer. Math. Soc. 28 (1971), 279-288.

InsttTUte for Medicine and Mathematics, Oho University, Athens, OHo 45701 